\title{
On the bisection operation
}

\author{
Sergio Cesare Masin and Enrico Toffalini \\ University of Padua, Padua, Italy
}

For two perceived sensory magnitudes, $A$ and $B$, the bisection operation yields a perceived sensory magnitude, $C$, equally different from $A$ and $B$. Available data do not resolve whether this operation is equivalent to a linear or a nonlinear mean. We tested these alternatives, using the evidence of functional measurement research that ratings are linear sensory measures. With $A$ and $B$ varied factorially, linear means predict that the curves relating rated $C$ to rated $A$ for each $B$ are parallel straight lines, and nonlinear means predict that these curves are nonlinear and nonparallel. Using brightness and perceived size as sensory attributes, the present experiments confirm the first of these predictions, indicating that the bisection operation is equivalent to a linear mean.

Given two perceived magnitudes of a single kind of sensory attribute, $A$ and $B$, the operation of bisection is the selection of the perceived magnitude of this attribute, $C$, that differs from $A$ as much as it differs from $B$ (Plateau, 1872). With $M_{A}, M_{B}$, and $M_{C}$ denoting the measures of $A, B$, and $C$, respectively, Pfanzagl (1959) proposed that

$$
M_{C}=w \cdot M_{A}+(1-w) \cdot M_{B},
$$

with $w$ constant in the interval $[0,1]$, and showed that the bisection operation is compatible with this weighted arithmetic mean when it satisfies the following condition.

\section{Bisymmetry Condition}

For a given sensory attribute, let $M_{1}, M_{2}, M_{3}$, and $M_{4}$ denote any measures of perceived magnitude sufficiently different from one another. Throughout this article, subscript numerals are used as labels. Bisections yield the measures

$$
\begin{gathered}
M_{12}=w \cdot M_{1}+(1-w) \cdot M_{2} \\
M_{34}=w \cdot M_{3}+(1-w) \cdot M_{4} \\
M_{13}=w \cdot M_{1}+(1-w) \cdot M_{3} \\
M_{24}=w \cdot M_{2}+(1-w) \cdot M_{4} \\
M_{14}=w \cdot M_{1}+(1-w) \cdot M_{4} \\
M_{23}=w \cdot M_{2}+(1-w) \cdot M_{3} \\
M_{1234}=w \cdot M_{12}+(1-w) \cdot M_{34} \\
M_{1324}=w \cdot M_{13}+(1-w) \cdot M_{24} \\
M_{1423}=w \cdot M_{14}+(1-w) \cdot M_{23} .
\end{gathered}
$$

With $w$ unknown, Equations 2-5, 8, and 9 imply

$$
M_{1234}=M_{1324} \text {. }
$$

Equation 11 expresses the bisymmetry condition (Aczél, 1948).

When $w=.5$, Equations $2-10$ imply

$$
M_{1234}=M_{1324}=M_{1423} \text {. }
$$

The empirical verification of all equalities in Equation 12 is believed to support the possibility that $M_{C}-$ $M_{A}=M_{B}-M_{C}(w=.5)$, making actual measurement possible (Adams \& Fagot, 1975; Coombs, Dawes, \& Tversky, 1970; Cross, 1964; Fagot \& Stewart, 1970; Irtel, 2005; Pfanzagl, 1971; Raslear, Shurtleff, \& Simmons, 1992).

\section{Gage's Condition}

When the effects of the orders of presentation of $A, B$, and $C$ are counterbalanced, Cross $(1964$, p. 5) showed that the bisymmetry condition is equivalent to the following condition proposed by Gage (1934a). Let $M_{1}$ and $M_{2}$ denote measures of two perceived magnitudes delimiting a sufficiently large sensory interval. Bisections yield the measures

$$
\begin{gathered}
M_{12}=w \cdot M_{1}+(1-w) \cdot M_{2} \\
M_{112}=w \cdot M_{1}+(1-w) \cdot M_{12} \\
M_{122}=w \cdot M_{12}+(1-w) \cdot M_{2} \\
M_{112122}=w \cdot M_{112}+(1-w) \cdot M_{122} .
\end{gathered}
$$

Equations 13-16 imply

$$
\begin{aligned}
M_{112122}= & \left(3 w^{2}-2 w^{3}\right) \cdot M_{1} \\
& +\left[1-\left(3 w^{2}-2 w^{3}\right)\right] \cdot M_{2} .
\end{aligned}
$$


A comparison of Equation 17 with Equation 13 shows that

$$
M_{112122}=M_{12}
$$

when

$$
3 w^{2}-2 w^{3}=w .
$$

The nontrivial solution of Equation 19 is $w=.5$. Equation 18 expresses Gage's condition. Since $M_{C}-M_{A}=$ $M_{B}-M_{C}$ when $M_{112122}=M_{12}(w=.5)$, it is believed that any sufficiently large sensory interval can be successively bisected into equal intervals, any one of which can be used as the unit of sensory measurement (Cross, 1964; Fagot, 1961; Gage, 1934a; Newman, Volkmann, \& Stevens, 1937; Torgerson, 1958).

\section{Previous Tests}

Let $M$ denote any sensory measure and let $F$ denote the respective physical measure. An $F$ with subscripts denotes the physical measure relative to an $M$ with equal subscripts. One can test the equalities in Equation 12 by testing the equalities

$$
F_{1234}=F_{1324}=F_{1423}
$$

and can test the equality in Equation 18 by testing the equality

$$
F_{112122}=F_{12}
$$

when the same single process of information transformation generates each of the perceived magnitudes in the bisections defined by Equations $2-10$ and Equations 13-16 - that is, when the same single psychophysical function represents this transformation. ${ }^{1}$ In this case, when the measures in Equation 12 or Equation 18 are equal, the respective physical stimulus measures also are equal (Equation 20 or Equation 21).

Using 1 participant, Cross (1964) found that $F_{1234}=$ $F_{1324}$. Heller (2001) confirmed this result in 9 of 12 participants. With bisections produced by varying $C$ in ascending order, Gage (1934a, 1934b) found that $F_{112122}>F_{12}$ in all participants. With unconstrained variation of $C$ and with $A, B$, and $C$ presented in counterbalanced orders, Newman et al. (1937), Tommasi (1999), and Wolff (1935) found that $F_{112122}=F_{12}$ in all participants. Fagot and Stewart (1970) confirmed this result in 1 of 4 participants.

Heller (2001) and Fagot and Stewart (1970) had participants bisect brightness intervals, using three light stimuli presented simultaneously. Marks (1974, p. 252) noted that simultaneous light stimuli may involve differential effects of brightness contrast. For example, $F_{12}$ is associated with $F_{1}$ and $F_{2}$ when it produces $M_{12}$ (Equation 2) and is associated with $F_{1234}$ and $F_{34}$ when it reproduces $M_{12}$ (Equation 8). The brightness contrast due to $F_{1}$ and $F_{2}$ may differ from that due to $F_{1234}$ and $F_{34}$ (Leibowitz, Mote, \& Thurlow, 1953; Zavagno, Annan, \& Caputo, 2004). Thus, $F_{12}$ produces $M_{12}$ but may not reproduce $M_{12}$. That is, there may be two different psychophysical functions, one relating $F_{12}$ to $M_{12}$ (Equation 2) and the other relating $F_{12}$ to another sensory measure (Equation 8 ).
As was noted above, in this case, one cannot rigorously test the equalities in Equation 20, since the test demands that there be a single psychophysical function for all the bisections of the test. ${ }^{2}$ In the following experiment, a test of the equalities in Equation 20 was made that was an attempt to overcome this problem.

\section{EXPERIMENT 1}

\section{Method}

Participants. Twenty-eight undergraduate university students participated to fulfill a course requirement. They were not informed about the purpose of the experiment. Each participant served in two sessions, Sessions 1 and 2. Session 1 preceded Session 2.

Stimuli. On each trial, in each session, the participant made one bisection, using four successive stimuli, Stimuli 1, 2, 3, and 4 . The interstimulus interval was $1.5 \mathrm{sec}$. The duration of Stimuli 1-3 was $0.7 \mathrm{sec}$. Stimulus 4 was presented in the position of Stimulus 2 and remained visible until the trial was completed. Stimuli 1 and 3 produced the sensory interval to be bisected. Stimuli 2 and 4 served to bisect this interval. The participant increased the magnitude of Stimulus 4 by pressing the right of two keys juxtaposed horizontally and decreased it by pressing the left key. Viewing distance was about $0.5 \mathrm{~m}$.

Session 1. Stimuli 1-4 were white circles with a $0.25-\mathrm{mm}$ circumference concentric with a $320 \times 240 \mathrm{~mm}$ black background (HP L1720 display). The areas, $F_{1}, F_{2}, F_{3}$, and $F_{4}$, for Stimulus 1 or 3 were $1.77,9.62,54.11$, and $298.65 \mathrm{~cm}^{2}$, respectively. The diameter of Stimulus 4 varied in steps of $0.5 \mathrm{~mm}$. The illumination level was $35 \mathrm{~lx}$.

Session 2. Stimuli 1-4 were 12-mm achromatic squares. Stimuli 1-3 were horizontally aligned from left to right, in this order, in the middle of a $0.2-\mathrm{cd} / \mathrm{m}^{2} 320 \times 170 \mathrm{~mm}$ dark gray background (Apple Multiple Scan 1705 display). They were separated by a gap of $11 \mathrm{~mm}$. The luminances, $F_{1}, F_{2}, F_{3}$, and $F_{4}$, for Stimulus 1 or 3 were $1.4,5,20$, and $80 \mathrm{~cd} / \mathrm{m}^{2}$, respectively. Stimulus 1 was dimmer than Stimulus 3. The luminance of Stimulus 4 varied in discrete steps. A power function with an exponent of 2 related the number of these steps, counted from $1.4 \mathrm{~cd} / \mathrm{m}^{2}$, to the luminance of Stimulus 4 . The number of steps needed to reach $80 \mathrm{~cd} / \mathrm{m}^{2}$ was 63 . The illumination level was $0.1 \mathrm{~lx}$. Before the session started, the participant was adapted to this illumination for $5 \mathrm{~min}$.

Procedure. The sensory attribute used for bisection was perceived size in Session 1 and was brightness in Session 2. Except for the sensory attribute, the procedures for Sessions 1 and 2 were the same. Bisections were made by selecting a physical measure for Stimulus 2 as follows. On each trial, Stimuli 1-4 were initially presented successively once. In this initial presentation, Stimulus 2 was equal to Stimulus 1 or 3, and Stimulus 4 was equal to Stimulus 2. The participant was asked to vary Stimulus 4 until the difference in perceived size or brightness between Stimulus 4 and Stimulus 1 equaled that between Stimulus 4 and Stimulus 3. Bracketing was allowed. The resulting measure of Stimulus 4 was assigned to Stimulus 2. So that the participant could judge whether this new measure of Stimulus 2 produced a satisfactory bisection, Stimuli 1-4 were presented again successively as soon as the participant pressed a key. When the participant was not satisfied that Stimulus 2 produced the bisection, the participant varied Stimulus 4 again, finding another measure for Stimulus 2 that more precisely produced the bisection. Stimuli 1-4 were then presented again when the participant pressed a key, now with Stimulus 2 having this other measure. The participant repeated this process as many times as needed until the participant was satisfied that Stimulus 2 produced the bisection.

The participant made 4 bisections for each of the six different combinations of $F_{1}, F_{2}, F_{3}$, and $F_{4}$ for Stimulus 1 with $F_{1}, F_{2}, F_{3}$, and $F_{4}$ for Stimulus 3 . For each of these combinations, Stimuli 1-3 appeared in one order for 2 bisections and in the reversed order for 
the other 2. For each of these two sets of 2 bisections, Stimulus 2 was initially equal to Stimulus 1 for 1 bisection and to Stimulus 3 for the other. The resulting 24 bisections were made in random order.

For each of the mentioned six combinations of $F_{1}, F_{2}, F_{3}$, and $F_{4}$, the mean of the respective four physical measures of Stimulus 2 was calculated. These means were $F_{12}, F_{34}, F_{13}, F_{24}, F_{14}$, and $F_{23}$ (Equations $2-7)$. Using the counterbalancing conditions previously described, the participant made 4 bisections for $F_{12}$ and $F_{34}, 4$ for $F_{13}$ and $F_{24}$, and 4 for $F_{14}$ and $F_{23}$, producing the mean physical measures of Stimulus 2, $F_{1234}, F_{1324}$, and $F_{1423}$ (Equations $8-10$ ). These 12 bisections were made in random order. The total of 36 bisections were carried out with no noticeable interruption between bisections.

\section{Results and Discussion}

For size and brightness, Table 1 reports $F_{12}, F_{34}, F_{13}$, $F_{24}, F_{14}$, and $F_{23}$, and Table 2 reports $F_{1234}, F_{1324}$, and $F_{1423}$ for each participant. Separate ANOVAs were used to determine the statistical significance of differences between $F_{1234}, F_{1324}$, and $F_{1423}$. Boldface type is used in Table 2 to indicate significance. Consider each participant for either size or brightness. When they are printed in normal type, all three means did not differ significantly from each other. When only one mean is printed in boldface type, it differed significantly from one or both of the other two. When only two means are printed in boldface type, they differed significantly from each other but not from the remaining mean. Finally, when all three means are printed in boldface type, they were all significantly different from each other.

The results in Table 2 support the possibility that $M_{1234}=M_{1324}=M_{1423}$. In each participant, $F_{1234}, F_{1324}$, and $F_{1423}$ did not differ significantly from each other at least once, for size or brightness. With individual $F_{1234}$, $F_{1324}$, and $F_{1423}$ as scores, an ANOVA showed that the means of $F_{1234}, F_{1324}$, and $F_{1423}$ did not differ significantly from each other, for size and brightness $[F(2,54)=0.9$ and $F(2,54)=1.5$, respectively].

Bisections made in the first session did not affect those made in the second session. Correlations of $F_{1234}, F_{1324}$, and $F_{1423}$ for size with $F_{1234}, F_{1324}$, or $F_{1423}$ for brightness ranged from -.09 to .09 . None was statistically different from zero. Correlations between $F_{1234}, F_{1324}$, and $F_{1423}$ for size and between $F_{1234}, F_{1324}$, and $F_{1423}$ for brightness ranged from .63 to .79 . Each differed statistically from zero at the .01 level.

Inspection of Table 2 shows individual differences. Such individual differences are expected to occur even when $w=.5$, since the exponents of the psychophysical power function for circle size (Griffin, 1985; Rule, 1966) and brightness (Marks \& Stevens, 1966) differ individually. That is, with $F_{1}, F_{2}, F_{3}$, and $F_{4}$ being the same for each participant, different exponents involve that $F_{1234}$, $F_{1324}$, and $F_{1423}$ differ individually if bisections produce equidifferent perceived magnitudes $(w=.5)$.

Insufficiency of the bisymmetry and Gage conditions. The bisection operation empirically satisfied the equalities in Equation $12(w=.5)$. Given this result, this operation is believed to produce equal sensory intervals (Coombs et al., 1970; Cross, 1964; Fagot, 1961; Fagot \& Stewart, 1970; Falmagne, 1974; Gage, 1934a; Kristof, 1968; Newman et al., 1937; Pfanzagl, 1971; Raslear,

Table 1

$F_{12}, F_{34}, F_{13}, F_{24}, F_{14}$, and $F_{23}$ for Size and Brightness, for Each of 28 Participants in Experiment 1

\begin{tabular}{|c|c|c|c|c|c|c|c|c|c|c|c|c|}
\hline \multirow[b]{2}{*}{ Participant } & \multicolumn{6}{|c|}{ Area $\left(\mathrm{cm}^{2}\right)$} & \multicolumn{6}{|c|}{ Luminance $\left(\mathrm{cd} / \mathrm{m}^{2}\right)$} \\
\hline & $F_{12}$ & $F_{34}$ & $F_{13}$ & $F_{24}$ & $F_{14}$ & $F_{23}$ & $F_{12}$ & $F_{34}$ & $F_{13}$ & $F_{24}$ & $F_{14}$ & $F_{23}$ \\
\hline 1 & 4.2 & 142.3 & 13.5 & 100.1 & 92.0 & 22.2 & 3.3 & 41.5 & 6.2 & 18.7 & 13.3 & 11.7 \\
\hline 2 & 3.9 & 140.3 & 18.1 & 98.0 & 97.3 & 22.1 & 3.5 & 31.5 & 8.2 & 17.9 & 17.6 & 10.4 \\
\hline 3 & 4.2 & 157.2 & 14.8 & 109.1 & 81.8 & 23.0 & 2.7 & 40.4 & 6.4 & 17.6 & 12.2 & 8.4 \\
\hline 4 & 4.4 & 141.8 & 18.5 & 101.2 & 77.5 & 27.5 & 2.7 & 46.5 & 7.1 & 26.6 & 15.3 & 10.9 \\
\hline 5 & 4.7 & 143.8 & 15.9 & 108.0 & 83.6 & 23.3 & 3.6 & 39.7 & 8.0 & 25.1 & 21.5 & 11.7 \\
\hline 6 & 4.4 & 120.6 & 19.6 & 101.8 & 88.2 & 24.0 & 2.8 & 32.6 & 7.3 & 15.8 & 12.0 & 10.4 \\
\hline 7 & 5.0 & 136.3 & 16.8 & 89.3 & 65.6 & 27.2 & 3.2 & 38.9 & 6.1 & 17.9 & 16.5 & 9.2 \\
\hline 8 & 4.4 & 150.7 & 11.9 & 105.9 & 85.5 & 23.8 & 3.2 & 35.3 & 6.6 & 26.6 & 12.4 & 12.0 \\
\hline 9 & 4.4 & 131.2 & 16.0 & 91.7 & 72.6 & 22.6 & 2.8 & 43.8 & 7.8 & 22.9 & 24.5 & 11.7 \\
\hline 10 & 4.6 & 157.2 & 14.8 & 139.5 & 140.4 & 23.1 & 3.0 & 29.2 & 6.6 & 12.9 & 10.4 & 8.8 \\
\hline 11 & 4.5 & 163.3 & 20.0 & 114.9 & 102.6 & 22.3 & 3.3 & 38.6 & 6.2 & 16.3 & 11.1 & 10.9 \\
\hline 12 & 4.5 & 138.8 & 17.4 & 88.7 & 73.3 & 22.4 & 3.2 & 39.7 & 8.6 & 21.2 & 11.7 & 13.8 \\
\hline 13 & 4.6 & 155.9 & 16.5 & 110.9 & 92.6 & 28.0 & 3.5 & 41.1 & 8.0 & 30.5 & 24.8 & 12.6 \\
\hline 14 & 4.0 & 144.8 & 13.1 & 93.1 & 80.5 & 23.2 & 2.6 & 36.0 & 6.4 & 18.4 & 8.0 & 10.4 \\
\hline 15 & 4.2 & 168.4 & 15.1 & 109.8 & 80.3 & 25.3 & 3.1 & 36.8 & 6.9 & 17.9 & 13.3 & 10.6 \\
\hline 16 & 4.7 & 135.6 & 17.7 & 105.9 & 64.4 & 26.1 & 3.1 & 33.6 & 8.6 & 16.8 & 11.5 & 11.3 \\
\hline 17 & 4.2 & 124.3 & 15.4 & 84.0 & 72.1 & 21.7 & 3.0 & 25.4 & 7.1 & 15.8 & 11.1 & 10.9 \\
\hline 18 & 4.2 & 148.3 & 19.9 & 101.4 & 87.0 & 26.6 & 3.2 & 43.0 & 6.6 & 17.6 & 9.4 & 9.2 \\
\hline 19 & 4.6 & 134.1 & 15.4 & 90.9 & 63.6 & 23.6 & 3.1 & 33.6 & 6.4 & 16.5 & 12.6 & 11.1 \\
\hline 20 & 4.6 & 140.5 & 15.7 & 110.9 & 89.6 & 20.9 & 3.3 & 25.1 & 7.1 & 11.3 & 10.0 & 9.4 \\
\hline 21 & 4.2 & 150.1 & 17.0 & 131.2 & 106.0 & 25.3 & 3.1 & 31.2 & 6.8 & 12.6 & 7.5 & 9.6 \\
\hline 22 & 4.6 & 141.8 & 18.4 & 103.3 & 104.3 & 26.9 & 3.1 & 32.6 & 9.6 & 21.2 & 13.6 & 9.6 \\
\hline 23 & 4.1 & 136.3 & 13.7 & 78.0 & 69.4 & 21.5 & 4.6 & 32.6 & 8.2 & 21.2 & 10.4 & 11.3 \\
\hline 24 & 3.7 & 128.8 & 16.4 & 92.9 & 75.3 & 24.8 & 3.1 & 40.0 & 6.4 & 25.4 & 21.2 & 9.6 \\
\hline 25 & 4.8 & 129.3 & 17.4 & 99.3 & 116.6 & 29.7 & 3.5 & 37.8 & 6.1 & 16.3 & 7.7 & 9.8 \\
\hline 26 & 4.9 & 170.9 & 20.8 & 140.0 & 97.7 & 23.3 & 3.3 & 35.7 & 7.8 & 25.1 & 14.3 & 13.1 \\
\hline 27 & 4.3 & 139.5 & 21.5 & 100.8 & 88.4 & 25.2 & 3.3 & 46.1 & 8.0 & 29.9 & 25.7 & 13.3 \\
\hline 28 & 5.8 & 155.6 & 18.2 & 108.9 & 92.8 & 26.8 & 3.1 & 42.3 & 8.6 & 24.1 & 19.5 & 11.5 \\
\hline Grand mean & 4.5 & 143.8 & 16.8 & 103.9 & 87.2 & 24.4 & 3.2 & 36.8 & 7.3 & 20.0 & 14.3 & 10.8 \\
\hline
\end{tabular}


Table 2

$F_{1234}, F_{1324}$, and $F_{1423}$ (Equation 20; With Standard Errors) for Size and Brightness for Each of 28 Participants in Experiment 1

\begin{tabular}{|c|c|c|c|c|c|c|c|c|c|c|c|c|}
\hline \multirow[b]{2}{*}{ Participant } & \multicolumn{6}{|c|}{ Area $\left(\mathrm{cm}^{2}\right)$} & \multicolumn{6}{|c|}{ Luminance $\left(\mathrm{cd} / \mathrm{m}^{2}\right)$} \\
\hline & $F_{1234}$ & $S E$ & $F_{1324}$ & $S E$ & $F_{1423}$ & $S E$ & $F_{1234}$ & $S E$ & $F_{1324}$ & $S E$ & $F_{1423}$ & $S E$ \\
\hline 1 & 43.8 & 3.7 & 49.7 & 4.6 & 51.3 & 1.7 & 8.3 & 0.5 & 11.1 & 1.2 & 11.2 & 0.3 \\
\hline 2 & 55.8 & 0.5 & 39.8 & 2.2 & 44.0 & 2.7 & 11.7 & 3.9 & 11.7 & 2.2 & 13.6 & 1.7 \\
\hline 3 & 51.4 & 3.1 & 49.6 & 2.8 & 45.2 & 1.2 & 10.1 & 0.2 & 9.5 & 0.2 & 9.0 & 0.5 \\
\hline 4 & 43.0 & 3.1 & 47.4 & 1.1 & 47.0 & 2.4 & 12.8 & 2.2 & 15.0 & 2.4 & 13.9 & 0.3 \\
\hline 5 & 46.4 & 3.7 & 53.4 & 3.6 & 47.4 & 1.2 & 18.4 & 1.3 & 15.5 & 0.7 & 16.3 & 0.5 \\
\hline 6 & 39.2 & 3.7 & 46.4 & 3.5 & 41.6 & 1.9 & 10.0 & 0.9 & 11.2 & 0.8 & 14.9 & 1.2 \\
\hline 7 & 38.4 & 7.7 & 42.6 & 6.7 & 41.8 & 2.9 & 13.0 & 2.0 & 9.2 & 0.3 & 11.1 & 0.4 \\
\hline 8 & 51.0 & 2.2 & 48.3 & 2.2 & 45.4 & 3.3 & 11.6 & 0.5 & 12.8 & 0.6 & 11.7 & 0.1 \\
\hline 9 & 45.1 & 5.5 & 39.1 & 3.1 & 43.0 & 1.9 & 13.2 & 1.8 & 14.3 & 0.9 & 18.9 & 1.6 \\
\hline 10 & 59.5 & 3.5 & 56.9 & 3.3 & 41.9 & 21.0 & 7.4 & 0.9 & 7.9 & 0.3 & 10.0 & 0.7 \\
\hline 11 & 57.2 & 11.0 & 60.2 & 6.7 & 51.9 & 3.3 & 11.0 & 1.0 & 9.7 & 0.2 & 10.1 & 0.1 \\
\hline 12 & 44.4 & 2.1 & 42.0 & 1.5 & 43.4 & 1.7 & 10.8 & 0.4 & 14.5 & 0.5 & 12.8 & 0.6 \\
\hline 13 & 52.5 & 5.0 & 48.7 & 3.6 & 50.4 & 3.7 & 15.2 & 0.9 & 17.2 & 1.4 & 16.2 & 1.8 \\
\hline 14 & 41.2 & 1.5 & 40.6 & 4.2 & 46.6 & 2.1 & 8.6 & 0.4 & 10.0 & 0.1 & 8.2 & 0.6 \\
\hline 15 & 58.3 & 4.9 & 52.6 & 3.7 & 45.9 & 2.5 & 10.6 & 1.0 & 10.3 & 0.7 & 12.6 & 0.6 \\
\hline 16 & 38.4 & 1.1 & 48.8 & 2.7 & 41.4 & 2.2 & 10.3 & 1.8 & 12.6 & 0.7 & 11.6 & 0.2 \\
\hline 17 & 32.3 & 3.3 & 34.5 & 2.0 & 35.6 & 1.2 & 10.3 & 1.3 & 11.5 & 0.9 & 9.7 & 0.2 \\
\hline 18 & 57.1 & 1.7 & 54.6 & 1.0 & 52.5 & 1.7 & 13.3 & 1.6 & 11.3 & 0.6 & 9.3 & 0.1 \\
\hline 19 & 37.2 & 3.5 & 39.6 & 6.7 & 38.3 & 2.1 & 10.3 & 0.5 & 10.1 & 0.3 & 12.1 & 0.7 \\
\hline 20 & 51.6 & 5.4 & 47.6 & 2.4 & 41.8 & 3.9 & 8.7 & 0.8 & 8.1 & 0.2 & 9.3 & 0.3 \\
\hline 21 & 57.8 & 2.5 & 55.7 & 3.0 & 51.6 & 3.6 & 8.2 & 0.4 & 9.1 & 0.5 & 8.3 & 0.8 \\
\hline 22 & 50.6 & 4.5 & 51.2 & 2.7 & 57.0 & 3.1 & 12.4 & 0.6 & 14.4 & 0.8 & 11.3 & 0.3 \\
\hline 23 & 31.1 & 4.5 & 35.3 & 5.2 & 36.1 & 1.9 & 11.2 & 1.6 & 15.0 & 0.1 & 11.1 & 0.4 \\
\hline 24 & 40.0 & 2.6 & 42.9 & 2.1 & 43.3 & 1.1 & 13.2 & 1.4 & 12.0 & 0.8 & 13.9 & 1.4 \\
\hline 25 & 49.0 & 2.5 & 50.4 & 3.7 & 68.4 & 2.4 & 10.0 & 1.2 & 9.4 & 0.5 & 8.7 & 0.5 \\
\hline 26 & 63.8 & 2.0 & 66.3 & 2.9 & 54.6 & 2.2 & 13.0 & 1.1 & 14.4 & 0.5 & 14.2 & 0.6 \\
\hline 27 & 47.4 & 5.0 & 52.6 & 1.4 & 47.4 & 2.3 & 17.2 & 1.5 & 19.1 & 0.5 & 20.5 & 1.0 \\
\hline 28 & 59.3 & 6.1 & 52.7 & 1.8 & 53.1 & 1.7 & 16.9 & 1.0 & 14.8 & 1.5 & 15.0 & 1.1 \\
\hline Grand mean & 48.0 & 1.7 & 48.2 & 1.4 & 46.7 & 1.3 & 11.7 & 0.5 & 12.2 & 0.5 & 12.3 & 0.6 \\
\hline
\end{tabular}

1983; Torgerson, 1958). The following discussion shows that this belief should be reconsidered.

Using the weighted arithmetic mean to relate $M_{A}, M_{B}$, and $M_{C}$ to each other is arbitrary. Equivalently, one could use the weighted Hölder mean,

$$
M_{C}=\left[u \cdot M_{A}^{s}+(1-u) \cdot M_{B}^{s}\right]^{\frac{1}{s}},
$$

with $u$ constant in the interval $[0,1]$ and $s$ any real number. The Hölder mean is the harmonic, arithmetic, or square root mean when $s$ is equal to $-1,1$, or 2 , respectively, and tends to the geometric mean when $s$ tends to 0 . When $s=1$, Equation 22 is Equation 1 with $w=u$. Equation 22 encodes an infinite number of weighted means, one for each $s$.

When one substitutes each of Equations 2-10 with Equation 22 one has

$$
\begin{aligned}
& M_{12}=\left[u \cdot M_{1}^{s}+(1-u) \cdot M_{2}^{s}\right]^{\frac{1}{s}} \\
& M_{34}=\left[u \cdot M_{3}^{s}+(1-u) \cdot M_{4}^{s}\right]^{\frac{1}{s}} \\
& M_{13}=\left[u \cdot M_{1}^{s}+(1-u) \cdot M_{3}^{s}\right]^{\frac{1}{s}} \\
& M_{24}=\left[u \cdot M_{2}^{s}+(1-u) \cdot M_{4}^{s}\right]^{\frac{1}{s}}
\end{aligned}
$$

$$
\begin{aligned}
M_{14} & =\left[u \cdot M_{1}^{s}+(1-u) \cdot M_{4}^{s}\right]^{\frac{1}{s}} \\
M_{23} & =\left[u \cdot M_{2}^{s}+(1-u) \cdot M_{3}^{s}\right]^{\frac{1}{s}} \\
M_{1234} & =\left[u \cdot M_{12}^{s}+(1-u) \cdot M_{34}^{s}\right]^{\frac{1}{s}} \\
M_{1324} & =\left[u \cdot M_{13}^{s}+(1-u) \cdot M_{24}^{s}\right]^{\frac{1}{s}} \\
M_{1423} & =\left[u \cdot M_{14}^{s}+(1-u) \cdot M_{23}^{s}\right]^{\frac{1}{s}} .
\end{aligned}
$$

With $u$ unknown, Equations 23-26, 29, and 30 imply $M_{1234}=M_{1324}$ (Equation 11). With $u=.5$, Equations 23-31 imply $M_{1234}=M_{1324}=M_{1423}$ (Equation 12). Thus, all the infinite weighted means encoded in Equation 22 imply the bisymmetry condition.

When one substitutes each of Equations 13-16 with Equation 22, one has

$$
\begin{aligned}
& M_{12}=\left[u \cdot M_{1}^{s}+(1-u) \cdot M_{2}^{s}\right]^{\frac{1}{s}} \\
& M_{112}=\left[u \cdot M_{1}^{s}+(1-u) \cdot M_{12}^{s}\right]^{\frac{1}{s}} \\
& M_{122}=\left[u \cdot M_{12}^{s}+(1-u) \cdot M_{2}^{s}\right]^{\frac{1}{s}}
\end{aligned}
$$




$$
M_{112122}=\left[u \cdot M_{112}^{s}+(1-u) \cdot M_{122}^{s}\right]^{\frac{1}{s}} .
$$

Equations 32-35 imply

$$
\begin{aligned}
M_{112122}=\{ & \left(3 u^{2}-2 u^{3}\right) \cdot M_{1}^{s} \\
& \left.+\left[1-\left(3 u^{2}-2 u^{3}\right)\right] \cdot M_{2}^{s}\right\}^{\frac{1}{s}} .
\end{aligned}
$$

Comparison of Equation 36 with Equation 32 shows that $M_{112122}=M_{12}$ when $u=.5$. Thus, all the infinite weighted means encoded in Equation 22 also imply Gage's condition. This finding agrees with the consideration that the Gage and bisymmetry conditions are equivalent when order effects are removed (Cross, 1964).

Since the bisection operation is compatible with each of the means encoded in Equation 22, ${ }^{3}$ the testing of the bisymmetry condition does not allow determining which mean describes the actual bisection operation. At this stage of the inquiry, this conclusion indicates that this determination requires information external to the bisection operation.

Previous tests. Carterette and Anderson (1979) and Weiss (1975) tested Equation 1 as a descriptor of the actual bisection operation. Participants made bisections for each of some factorial combinations of $F_{A}$ and $F_{B}$. Equation 1 predicts that a straight line relates $M_{C}$ to $M_{A}$ for each $M_{B}$ and that these lines are parallel. A psychophysical function should exist that monotonically transforms $F_{A}, F_{B}$, and $F_{C}$ to $M_{A}, M_{B}$, and $M_{C}$, respectively, so that the curves relating $M_{C}$ to $M_{A}$ are parallel straight lines. The numerical determination of this psychophysical function was found possible for loudness and grayness. This possibility was interpreted to support that Equation 1 adequately describes the bisection operation. The authors above did not test other potentially equivalent descriptors of the bisection operation, such as the nonlinear means encoded in Equation 22.

Linearity of the response function. Thus, information external to the bisection operation is needed to determine whether a linear or a nonlinear weighted mean adequately describes this operation. Research using functional measurement has provided such external information in the finding that ratings are linear sensory measures (Anderson, 1962, 1974, 1981, 1982, 1996, 2001, 2007, 2008; Weiss, 2006). Before we proceed, we will provide a brief overview of this method of measurement.

Functional measurement allows determining the mathematical form of psychological laws by a factorial experimental design combined with a method of subjective evaluation applied to a single dependent factor. Consider a psychological law in which two independent factors determine a dependent factor. Let $M_{Z}$ be the measure of the dependent factor and $M_{X}$ and $M_{Y}$ those of the independent factors. For each factorial combination of $M_{X}$ and $M_{Y}$, the participant subjectively evaluates the dependent factor. Typically, this evaluation is a rating, $R_{Z}$. One factorial curve relates $R_{Z}$ to $M_{X}$ for each $M_{Y}$.

The response function relates $R_{Z}$ to $M_{Z}$. This function and the psychological law determine the pattern of facto- rial curves. Knowing the response function allows deducing the form of the psychological law from the factorial pattern. This deduction is straightforward when the response function is linear- that is, when

$$
R_{Z}=c_{0}+c_{1} M_{Z},
$$

with $c_{0}$ and $c_{1}$ constant. For example, one deduces that the psychological law is additive when the factorial curves are parallel and that this law is multiplicative when the factorial curves fan out as $M_{X}$ increases. $^{4}$

A basic question is whether the response function is linear. Thurstone (1929) found that end effects and relative stimulus frequency may bias ratings. Different classes of empirical evidence show that the response function is linear when one removes end effects by using two end anchors and the frequency effect by using graphic rating or numerical rating with a large number of response categories (Anderson, 1982; Parducci, 1982). A summary of nine of these classes of evidence is provided in Anderson (1996, pp. 94-98). Here, we exemplify the class of evidence that the linearity of the response function agrees with physical law (Anderson, 1983).

Two laws of physical friction apply to a flat object sliding on a horizontal board. Suppose the object and board are covered with sandpaper. One law is additive. It states that, for each combination of fixed grit numbers of the sandpapers of the object and board, the minimum physical force necessary to slide the object on the board depends additively on the two grit numbers. The other law is multiplicative. It states that this minimum force depends multiplicatively on the friction coefficient and the weight of the object. It is assumed that participants know both of these laws intuitively from extensive experience in the physical world.

Corneli and Vicovaro (2007) tested the additive law as follows. With the grit numbers of the object and board varied factorially, participants rated the amount of friction of the object on the board after they had felt with their fingers how rough the surfaces of the object and board were. If the response function is linear, the factorial curves relating rated friction to grit number of the object for each grit number of the board must be parallel. This prediction was confirmed empirically.

Corneli and Vicovaro (2007) tested the multiplicative law as follows. With the weight of the object and the grit number of the board varied factorially, the participants rated the amount of friction of the object on the board after they had hefted the object and felt how rough the surface of the board was. If the response function is linear, the factorial curves relating rated friction to object weight for each grit number of board must form a linear fan of curves. This prediction was also confirmed empirically.

\section{EXPERIMENT 2}

Equation 1 and Equation 22 with $s$ different from 1 make different predictions. Equation 1 predicts that the curves relating $M_{C}$ to $M_{A}$ for each $M_{B}$ are parallel straight lines, whereas Equation 22 with $s$ different from 1 predicts 
that these curves are nonlinear and nonparallel. The following experiment served to test these predictions.

Let $R$ be the mean rating $R_{A}, R_{B}$, or $R_{C}$ of $A, B$, or $C$, and let $M$ be the corresponding measure $M_{A}, M_{B}$, or $M_{C}$, respectively. Since $R=c_{0}+c_{1} M$, Equation 1 implies

$$
R_{C}=w \cdot R_{A}+(1-w) \cdot R_{B},
$$

and Equation 22 implies

$$
R_{C}=c_{0}+\left[u \cdot\left(R_{A}-c_{0}\right)^{s}+(1-u) \cdot\left(R_{B}-c_{0}\right)^{s}\right]^{\frac{1}{s}} .
$$

Equation 38 predicts that the curves relating $R_{C}$ to $R_{A}$ for each fixed $R_{B}$ are parallel straight lines, and Equation 39 predicts that these curves are nonlinear and nonparallel.

\section{Method}

Participants. Twenty undergraduate university students participated for pay. They were not informed about the purpose of the experiment. None of them had participated in Experiment 1. Two different groups of 10 participants were formed, Groups 1 and 2. There were two sessions for each group, Sessions 1 and 2. Session 1 preceded Session 2.

Stimuli and Procedure for Group 1. In Session 1, the participant bisected intervals of perceived size. The bisection procedure, the stimuli, and the stimulus presentation and counterbalancing conditions were the same as those used for Session 1 of Experiment 1, with the following exceptions. The area for Stimulus $1, F_{A}$, was 4.34, 17.35, 70.88 , or $283.53 \mathrm{~cm}^{2}$, and that for Stimulus $3, F_{B}$, was 1.04, 9.90, 39.59, or $159.48 \mathrm{~cm}^{2}$. Each $F_{A}$ was combined with each $F_{B}$. For each of these 16 combinations, the participant made four bisections, each producing one area of Stimulus 2. The mean of these four areas was $F_{C}$.

In Session 2, the participant rated the circle sizes corresponding to $F_{A}, F_{B}$, and $F_{C}$. The series of these 24 sizes was presented five times consecutively, each time with sizes in random order. Stimuli 1-3 were presented with the same presentation conditions as those in the first session. Stimulus 4 was not presented. Stimuli 1 and 2 were used as end anchors. Their area was 0.05 and $401 \mathrm{~cm}^{2}$, respectively. The area of Stimulus 3 was $F_{A}, F_{B}$, or $F_{C}$. The participant rated the size of Stimulus 3 using the integers from 1 to 100 , with 1 defined as the rating for the smaller anchor and 100 as that for the larger anchor.
Stimuli and Procedure for Group 2. In Session 1, the participant bisected brightness intervals. The bisection procedure, the stimuli, and the stimulus presentation and counterbalancing conditions were the same as those used for Session 2 of Experiment 1, with the following exceptions. The luminance for Stimulus $1, F_{A}$, was $4,10,20$, or $34 \mathrm{~cd} / \mathrm{m}^{2}$, and that for Stimulus $3, F_{B}$, was 7,15 , 27 , or $43 \mathrm{~cd} / \mathrm{m}^{2}$. When $F_{A}>F_{B}$, Stimuli 1 and 3 were switched in position so that the luminance of the left square was less than that of the right square on all trials. Each $F_{A}$ was combined with each $F_{B}$. For each of these 16 combinations, the participant made four bisections, each producing one luminance of Stimulus 2. The mean of these four luminances was $F_{C}$.

In Session 2, the participant rated each of the brightnesses corresponding to $F_{A}, F_{B}$, and $F_{C}$. The series of these 24 brightnesses was presented five times consecutively, each time with brightnesses in random order. Stimuli 1-3 were presented with the same presentation conditions as those in the first session. Stimuli 1 and 2 were the end anchors. Their luminance was 1.4 and $70 \mathrm{~cd} / \mathrm{m}^{2}$, respectively. The luminance of Stimulus 3 was $F_{A}, F_{B}$, or $F_{C}$. The participant rated the brightness of Stimulus 3 using the integers from 1 to 100 , with 1 being the rating for the dimmest anchor and 100 that for the brightest anchor.

\section{Results and Discussion}

In Figure 1, the left diagram reports mean rated size $R_{C}$ plotted against mean rated size $R_{A}$ for each mean rated size $R_{B}$. Factorial curves were practically parallel straight lines, as predicted by Equation 38 . This result empirically demonstrates that a linear mean adequately describes the bisection operation. The interaction and its components were not significant $\left[F(9,81)=0.8\right.$ and $F_{\mathbf{S}}(1,9)=0.07$ 2.2 , respectively].

In Figure 1, the right diagram reports mean rated brightness $R_{C}$ plotted against mean rated brightness $R_{A}$ for each mean rated brightness $R_{B}$. Factorial curves were practically parallel. The agreement with Equation 38 was partial, since factorial curves were slightly curved. The interaction was not significant $[F(9,81)=1.6]$. The quadratic-quadratic component of the interaction was significant $[F(1,9)=7.4, p<.05]$, with none of the re-
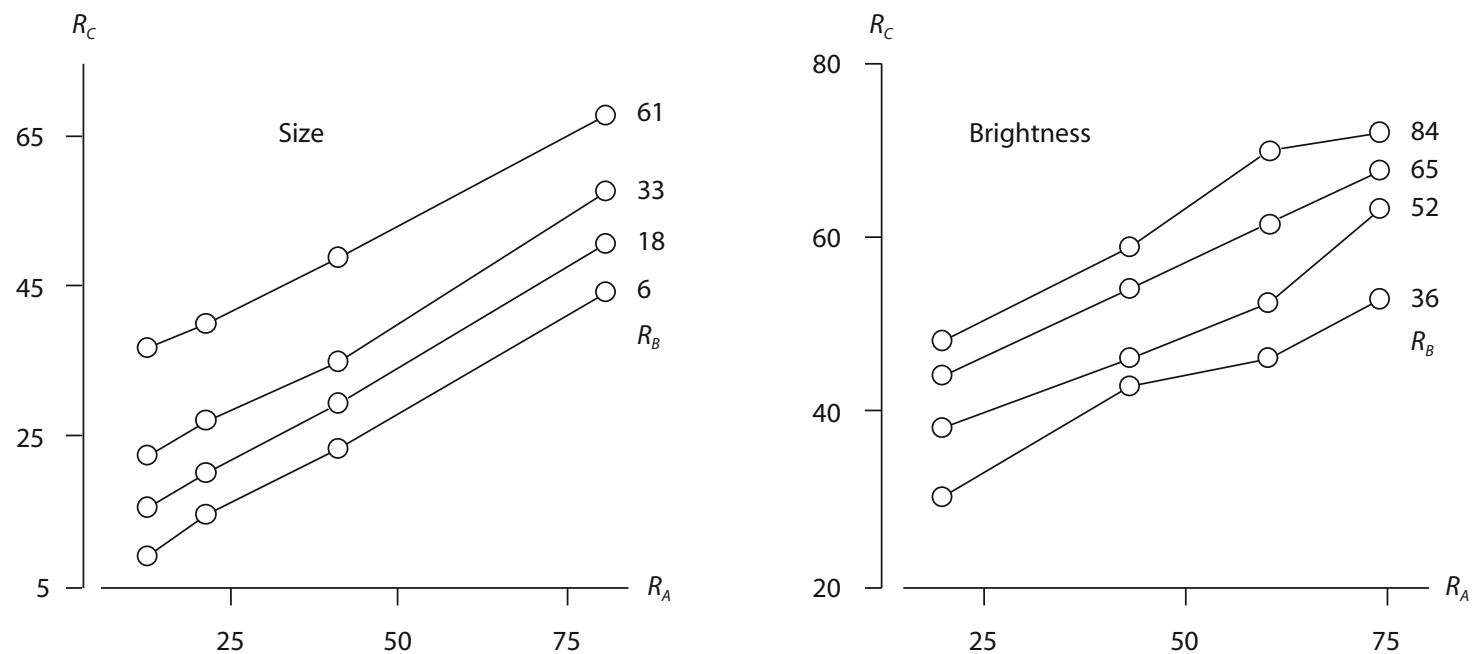

Figure 1. Mean rating, $R_{C}$, of the perceived magnitude (size or brightness) obtained by bisection plotted against the mean rating, $R_{A}$, of one of the two terminal perceived magnitudes used for the bisection, with the parameter being the mean rating, $R_{B}$, of the other terminal perceived magnitude. 
maining components of the interaction being significant $\left[F_{\mathrm{S}}(1,9)=0.04-4.3\right]$.

The following experiment served to explore the possibility that the variation of $w$ with $M_{A}$ and $M_{B}$ caused the quadratic-quadratic component of the interaction.

\section{EXPERIMENT 3}

Let $M_{1}$ and $M_{9}$ denote the measures of the terminal brightnesses of a large brightness interval. The participant was asked to make the bisections defined by the equations

$$
\begin{aligned}
& M_{5}=w \cdot M_{1}+(1-w) \cdot M_{9} \\
& M_{3}=w \cdot M_{1}+(1-w) \cdot M_{5} \\
& M_{7}=w \cdot M_{5}+(1-w) \cdot M_{9} \\
& M_{5}^{\prime}=w \cdot M_{3}+(1-w) \cdot M_{7} \\
& M_{2}=w \cdot M_{1}+(1-w) \cdot M_{3} \\
& M_{4}=w \cdot M_{3}+(1-w) \cdot M_{5} \\
& M_{6}=w \cdot M_{5}+(1-w) \cdot M_{7} \\
& M_{8}=w \cdot M_{7}+(1-w) \cdot M_{9} .
\end{aligned}
$$

Equation 43 defines the bisection needed to test Gage's condition. The brightnesses resulting from the bisections were subsequently rated by the participant. If $w$ in Equations $40-42$ and $44-47$ varies with the respective perceived magnitudes, these magnitudes must be unequally spaced, and thus, the respective mean ratings must be unequally spaced.

\section{Method}

Participants. Thirty-five undergraduate university students participated to fulfill a course requirement. They were not informed about the purpose of the experiment. None had participated in Experiment 1 or 2 . There were two sessions, Sessions 1 and 2. Session 1 preceded Session 2.

Stimuli and Procedure for Session 1. The bisection procedure, stimuli, and stimulus presentation and counterbalancing conditions were the same as those used for Session 2 of Experiment 1, with the following exceptions. With the luminances $F_{1}$ and $F_{9}$ being 5 and $45 \mathrm{~cd} / \mathrm{m}^{2}$, respectively, the participant made, 12 times consecutively, the bisection defined by Equation 40 (yielding the mean luminance $F_{5}$ ), then made, 8 times in random order, each of the bisections defined by Equations 41 and 42 (mean luminances $F_{3}$ and $F_{7}$, respectively), then made, 8 times consecutively, the bisection defined by Equation 43 (mean luminance $F_{5}^{\prime}$ ), and finally made, 4 times in random order, each of the bisections defined by Equations 44-47 (mean luminances $F_{2}, F_{4}, F_{6}$, and $F_{8}$, respectively).

Stimuli and Procedure for Session 2. The participant rated the brightnesses produced by the nine luminances $F_{1}-F_{9}$. The stimuli and presentation conditions and the procedure for the rating were the same as those used for Session 2 of Group 2 in Experiment 2, with the following exceptions. Stimuli 1 and 2, the end anchors, had luminances of 1.4 and $80 \mathrm{~cd} / \mathrm{m}^{2}$, respectively. The luminances for Stimulus 3 were $F_{1}-F_{9}$. These nine luminances were presented 10 times consecutively, each time in random order.

\section{Results and Discussion}

Figure 2 shows mean rated brightness, $R$, as a function of $M_{1}-M_{9}$ for 27 participants with $F_{5}^{\prime}=F_{5}[t \mathrm{~s}(18)=$ $0.01-1.9]$. One error bar above or below $R$ shows the standard error of $R$. The curve is a least-squares fourthdegree polynomial. The quartic trend of $R$ was significant $[F(1,26)=9.4, p<.005]$. For another 4 participants, $F_{5}^{\prime}>F_{5}[t \mathrm{~s}(18)=2.7-4.7, p<.05]$, and for the remaining $4, F_{5}^{\prime}<F_{5}[t \mathrm{~s}(18)=2.2-3.4, p<.05]$.

Ratings should not have caused the quartic trend of $R$, since ratings of brightness increase monotonically with luminance in the range of luminances used in the present study (Marks, 1968). Plausibly, this trend shows that $w$ varied with the two terminal brightnesses used for the bisection. The results indicate that $w$ was larger than .5 when both of these brightnesses were extreme. This effect was larger when the terminal brightnesses were largest. Accordingly, after removal from the analysis of the cases corresponding to $M_{8}$ and $M_{9}$, the linear trend of $R$ was significant, with higher order trends not reaching significance $\left[F(1,26)=241, p<.001\right.$, and $F_{\mathrm{S}}(1,26)=0.02-3.5$, respectively].

The test of Gage's condition was insensitive to small changes in the spacing of brightnesses. Deviations of $w$ from .5 were too small to make $F_{5}^{\prime}$ statistically different from $F_{5}$. The analysis of the trend of $R$ revealed small changes in the spacing of brightnesses, since it involved the comparison of several means, rather than of only two means, as in the test of Gage's condition.

In Figure 2, the results show that $w$ was very close to .5 in the bisections defined by Equation 40. Thus, this equation essentially states that

$$
M_{5}=\frac{M_{1}+M_{9}}{2} \text {. }
$$

Assuming that the psychophysical function is described by the power function

$$
M=a \cdot F^{n}+b,
$$

with $a, b$, and $n$ constant, Equations 48 and 49 imply

$$
F_{5}^{n}=\frac{F_{1}^{n}+F_{9}^{n}}{2}
$$

(Stefanini, 1888). With $F_{1}$ and $F_{9}$ fixed, one can estimate the exponent of the power function for each individual $F_{5}$

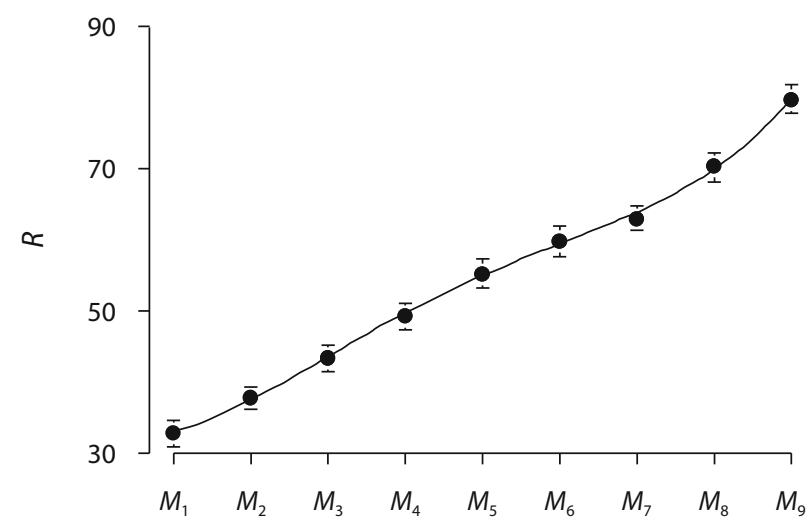

Figure 2. Mean rating, $R$, of brightness plotted against the corresponding $M$ of Equations 40-42 and 44-47. The curve depicts a fourth-degree polynomial. 
by determining $n$ numerically in Stefanini's Equation 50, excluding the trivial solution for $n=0$. The mean $n$ so found was 0.19. Using Stefanini's equation, Stewart, Fagot, and Eskildsen (1967) found a mean $n$ of 0.17 when the brightness interval used for bisection was about as wide as that considered here. These exponents are close to the mean $n$ of 0.21 obtained for brightness using ratings with a large number of response categories (Marks, 1968).

\section{CONCLUSION}

Equations 2-10 and 13-16 describe the bisection operation as being equivalent to a linear mean (Equation 1). In order for a linear mean to actually describe this operation, it is typically believed that the bisections defined by Equations $2-10$ must empirically satisfy the bisymmetry condition (Equation 11) and that those defined by Equations 13-16 must empirically satisfy Gage's condition (Equation 18). The results of Experiment 1 show that the participants performed bisections that empirically satisfied these conditions. However, it was subsequently demonstrated that the nonlinear means encoded in the weighted Hölder mean (Equation 22) also imply the bisymmetry and Gage conditions. This demonstration shows that tests of the bisymmetry and Gage conditions are insufficient to determine which mean adequately describes the actual bisection operation and thus indicates that, at this stage of the inquiry, information external to the bisection operation is needed for this determination. Functional measurement research has provided such external information in the repeated finding that ratings are linear measures of perceived magnitude. This linearity implies that one can rewrite Equations 1 and 22 in terms of ratings, as in Equations 38 and 39, respectively. When the perceived magnitudes delimiting a bisection interval are varied factorially, Equation 38 implies that factorial curves are parallel straight lines, whereas Equation 39 implies that factorial curves are nonlinear and nonparallel. The present experimental results confirm the implications of Equation 38, thus supporting the idea that the bisection operation is equivalent to a linear weighted mean.

\section{AUTHOR NOTE}

We thank Daniel Algom, Norman Henry Anderson, and Lawrence Edward Marks for useful comments. Correspondence concerning this article should be addressed to S. C. Masin, Department of General Psychology, University of Padua, Via Venezia 8, 35131 Padua, Italy (e-mail: scm@unipd.it).

\section{REFERENCES}

ACZÉL, J. (1948). On mean values. Bulletin of the American Mathematical Society, 54, 392-400.

Adams, E. W., \& Fagot, R. F. (1975). On the theory of biased bisection operations and their inverses. Journal of Mathematical Psychology, 12, 35-52.

ANDERSON, N. H. (1962). Application of an additive model to impression formation. Science, 138, 817-818.

ANDERSON, N. H. (1974). Information integration theory: A brief survey. In D. H. Krantz, R. C. Atkinson, R. D. Luce, \& P. Suppes (Eds.), Contemporary developments in mathematical psychology: Vol. 2. Measurement, psychophysics and neural information processing (pp. 236305). San Francisco: Freeman.
ANDERSON, N. H. (1981). Foundations of information integration theory. New York: Academic Press.

ANDERSON, N. H. (1982). Methods of information integration theory. New York: Academic Press.

ANDERSON, N. H. (1983). Intuitive physics: Understanding and learning of physical relations. In T. J. Tighe \& B. E. Shepp (Eds.), Perception, cognition, and development: Interactional analyses (pp. 231-265). Hillsdale, NJ: Erlbaum.

Anderson, N. H. (1996). A functional theory of cognition. Mahwah, NJ: Erlbaum.

Anderson, N. H. (2001). Empirical direction in design and analysis. Mahwah, NJ: Erlbaum.

ANDERson, N. H. (2007). Unified science based on psychological laws. Teorie \& Modelli, 12, 13-40.

Anderson, N. H. (2008). Unified social cognition. New York: Francis \& Taylor.

Anstis, S. M., Smith, D. R. R., \& Mather, G. (2000). Luminance processing in apparent motion, Vernier offset and stereoscopic depth. Vision Research, 40, 657-675.

Carterette, E. C., \& Anderson, N. H. (1979). Bisection of loudness. Perception \& Psychophysics, 26, 265-280.

Coombs, C. H., Dawes, R. M., \& Tversky, A. (1970). Mathematical psychology: An elementary introduction. Englewood Cliffs, NJ: Prentice Hall.

Corneli, E., \& Vicovaro, M. (2007). Intuitive cognitive algebra of sliding friction. Teorie \& Modelli, 12, 133-142.

Cross, D. V. (1964). An application of mean value theory to psychophysical measurement. In H. L. Lane (Ed.), Experimental analysis of the control of speech production and perception: VI (Rep. No. 6). Ann Arbor: University of Michigan Libraries.

DRÖSLER, J. (1995). The invariances of Weber's and other laws as determinants of psychophysical structures. In R. D. Luce, M. D'Zmura, D. Hoffman, G. J. Iverson, \& A. K. Romney (Eds.), Geometric representations of perceptual phenomena: Papers in honor of Tarow Indow on his 70th birthday (pp. 69-93). Mahwah, NJ: Erlbaum.

FAGOT, R. F. (1961). A model for equisection scaling. Behavioral Science, 6, 127-133.

Fagot, R. F., \& Stewart, M. R. (1970). Test of a response bias model of bisection. Perception \& Psychophysics, 7, 257-262.

FALMAGNE, J.-C. (1974). Foundations of Fechnerian psychophysics. In D. H. Krantz, R. C. Atkinson, R. D. Luce, \& P. Suppes (Eds.), Contemporary developments in mathematical psychology: Vol. 2. Measurement, psychophysics and neural information processing (pp. 127159). San Francisco: Freeman.

FALMAGNE, J.-C. (1985). Elements of psychophysical theory. New York: Oxford University Press.

GAGE, F. H. (1934a). An experimental investigation of the measurability of auditory sensation. Proceedings of the Royal Society B, 116, 103122.

GAGE, F. H. (1934b). An experimental investigation of the measurability of visual sensation. Proceedings of the Royal Society B, 116, 123138.

GrifFIN, T. L. C. (1985). Group and individual variations in judgment and their relevance to the scaling of graduated circles. Cartographica, 22, 21-37.

Heller, J. (2001). Mittenbildung bei achromatischen Farben: Das klassische Experiment von Plateau. Zeitschrift für Experimentelle Psychologie, 48, 259-271.

IrTel, H. (2005). Psychophysical scaling. In B. S. Everitt \& D. C. Howell (Eds.), Encyclopedia of statistics in behavioral science (Vol. 3, pp. 1628-1632). Chichester, U.K.: Wiley.

KRISTOF, W. (1968). Structural properties and measurement theory of certain sets admitting a concatenation operation. British Journal of Mathematical \& Statistical Psychology, 21, 201-229.

Leibowitz, H., Mote, F. A., \& ThurLow, W. R. (1953). Simultaneous contrast as a function of separation between test and inducing fields. Journal of Experimental Psychology, 46, 453-456.

MarKs, L. E. (1968). Stimulus range, number of categories, and form of the category-scale. American Journal of Psychology, 81, 467-479.

Marks, L. E. (1974). Sensory processes: The new psychophysics. New York: Academic Press.

Marks, L. E., \& Stevens, J. C. (1966). Individual brightness functions. Perception \& Psychophysics, 1, 17-24. 
Masin, S. C. (2007). Functional measurement in physics. Teorie \& Modelli, 12, 277-280.

Newman, E. B., Volkmann, J., \& Stevens, S. S. (1937). On the method of bisection and its relation to a loudness scale. American Journal of Psychology, 49, 134-137.

PARDUCCI, A. (1982). Category ratings: Still more contextual effects! In B. Wegener (Ed.), Social attitudes and psychophysical measurement (pp. 89-105). Hillsdale, NJ: Erlbaum.

Pfanzagl, J. (1959). A general theory of measurement applications to utility. Naval Research Logistics Quarterly, 6, 283-294.

Pfanzagl, J. (1971). Theory of measurement. Würzburg: PhysicaVerlag.

Plateau, J. (1872). Sur la mesure des sensations physiques, et sur la loi qui lie l'intensité de ces sensations à l'intensité de la cause excitante. Comptes Rendus Hebdomadaires des Séances de l'Académie des Sciences, 65, 677-680.

Raslear, T. G. (1983). A test of the Pfanzagl bisection model in rats. Journal of Experimental Psychology: Animal Behavior Processes, 9, 49-62.

Raslear, T. G., Shurtleff, D., \& Simmons, L. (1992). Loudness bisection and masking in the rat (Rattus norvegicus). Journal of Comparative Psychology, 106, 374-382.

RulE, S. J. (1966). Subject differences in exponents of psychophysical power functions. Perceptual \& Motor Skills, 23, 1125-1126.

STEFANINI, A. (1888). Sulla legge di oscillazione dei diapason e sulla misura della intensità del suono. Atti della Reale Accademia Lucchese di Scienze, Lettere ed Arti, 25, 305-400.

Stewart, M. R., Fagot, R. F., \& Eskildsen, P. R. (1967). Invariance tests for bisection and fractionation scaling. Perception \& Psychophysics, 2, 323-327.

Thurstone, L. L. (1929). Fechner's law and the method of equalappearing intervals. Journal of Experimental Psychology, 12, 214224

Tommasi, M. (1999). Experimental comparison of brightness judgments obtained by rating and bisection methods. Perceptual \& Motor Skills, 88, 903-909.
Torgerson, W. S. (1958). Theory and methods of scaling. New York: Wiley.

WEISs, D. J. (1975). Quantifying private events: A functional measurement analysis of equisection. Perception \& Psychophysics, 17, 351357.

WeIss, D. J. (2006). Analysis of variance and functional measurement: A practical guide. New York: Oxford University Press.

WolfF, W. (1935). Versuche zur Lautstärkeempfindung. Zeitschrift für Psychologie, 136, 325-340.

Zavagno, D., Annan, V., \& Caputo, G. (2004). The problem of being white: Testing the highest luminance rule. Vision, 16, 149-159.

\section{NOTES}

1. The tests are completely independent of the form of the psychophysical function, in accordance with the fact that each of Equations 1-18 is also completely independent of this form.

2. In brightness equisection, this problem is even more serious when two stimuli with fixed luminances and many aligned stimuli with intermediate luminances are presented simultaneously while the participant adjusts the intermediate luminances to make the brightnesses of all the stimuli equispaced perceptually (Anstis, Smith, \& Mather, 2000).

3. Falmagne (1985) and Drösler (1995) had already noted this compatibility for the arithmetic and geometric means.

4. Functional measurement also allows determining the mathematical form of physical laws. In this case, the factorial experimental design is combined with a method of physical measurement producing a ratio or interval scale of the dependent factor. No physical measure of the independent factors is needed. Functional measurement yields the physical scales of these independent factors. An example is provided in Masin (2007). Functional measurement may be useful in physics when the independent factors would be difficult to measure by customary physical methods.

(Manuscript received April 21, 2008; revision accepted for publication May 31, 2009.) 